\title{
Correction to: Major complications in extreme lateral interbody fusion access: multicentric study by Italian S.O.L.A.S. group
}

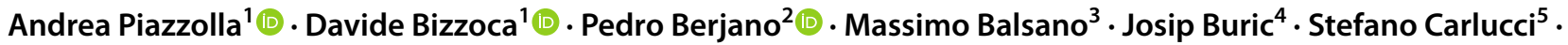 \\ Carlo Formica $^{2} \cdot$ Matteo Formica $^{6}$. Claudio Lamartina ${ }^{2} \cdot$ Corrado Musso $^{7} \cdot$ Francesco Tamburrelli $^{8}$. \\ Marco Damilano $^{2} \cdot$ Fulvio Tartara $^{9} \cdot$ Aldo Sinigaglia $^{2} \cdot$ Roberto Bassani $^{10} \cdot$ Massimiliano Neroni $^{11} \cdot$ Giovanni Casero $^{10}$. \\ Alessio Lovi ${ }^{11}$. Diego Garbossa ${ }^{12} \cdot$ Zullo Nicola $^{7} \cdot$ Biagio Moretti $^{1}$ (D)
}

Published online: 9 October 2020

(c) Springer-Verlag GmbH Germany, part of Springer Nature 2020

Correction to: European Spine Journal https://doi.org/10.1007/s00586-020-06542-0

Unfortunately, the 13th author name was incorrectly published in the original publication. The complete correct name should read as follow.

Fulvio Tartara

The original article has been corrected.

Publisher's Note Springer Nature remains neutral with regard to jurisdictional claims in published maps and institutional affiliations.

The original article can be found online at https://doi.org/10.1007/ s00586-020-06542-0.

Pedro Berjano

pberjano@gmail.com

1 School of Medicine, Department of Basic Medical Sciences, Neuroscience and Sense Organs, Orthopaedic, Trauma and Spine Unit, University of Bari "Aldo Moro"-AOU Policlinico Consorziale, Bari, Italy

2 IRCCS Istituto Ortopedico Galeazzi, Milan, Italy

3 Ortopedia e Traumatologia A, Centro Regionale Specializzato in Chirurgia Vertebrale, Azienda Ospedaliera Universitaria Integrata Verona (AOUI), Ospedale Borgo Trento, Verona, Italy

4 Villa Torri Hospital, Bologna, Italy

5 Department of Orthopaedics and Traumatology, SS Annunziata, Taranto, Italy
6 Clinica Ortopedica, IRCCS Azienda Ospedaliera Universitaria San Martino-IST, Genova, Italy

7 Unità Operativa Chirurgia della Colonna 2, Humanitas Gavazzeni, Bergamo, Italy

8 Department of Orthopedic Science and Traumatology. Spine Surgery Division, Catholic University Rome, Rome, Italy

9 Azienda Ospedaliera "Istituti Ospitalieri Di Cremona"-U.O.C. Di Neurochirurgia, Cremona, Italy

10 Salvator Mundi International Hospital, Rome, Italy

11 GSpine 3, IRCCS Istituto Ortopedico Galeazzi, Milan, Italy

12 Clinica Neurochirurgica, Città della Salute e della Scienza, "Molinette", Turin, Italy 